\title{
A note on involution rings
}

\author{
D. I. C. Mendes
}




\title{
A NOTE ON INVOLUTION RINGS
}

\author{
D. I. C. MENDES
}

Received 29 January, 2009

\begin{abstract}
Classifications of subdirectly irreducible involution rings via some properties of trace elements are given. As an application, von Neumann regular involution rings with central idempotent norm elements are described. The structure of certain involution rings in which the norms are multiplicatively generated by nilpotents is also determined.
\end{abstract}

2000 Mathematics Subject Classification: 16N60, 16W10

Keywords: involution ring, subdirectly irreducible ring, von Neumann regular ring, norm, trace

\section{INTRODUCTION}

All rings considered are associative. Let us recall that an involution ring $R$ is a ring with an additional unary operation $*$, called involution, such that $(a+b)^{*}=$ $a^{*}+b^{*},(a b)^{*}=b^{*} a^{*}$, and $\left(a^{*}\right)^{*}=a$ for all $a, b \in R$. An element of an involution ring $R$ which is either symmetric or skew-symmetric shall be called a $*$-element and a $*$-ideal $I$ of $R$ is an ideal of $R$ which is closed under involution, that is, $I^{*}=$ $\left\{a^{*} \in R: a \in I\right\} \subseteq I$. A subring $B$ of $R$ such that $B A B \subseteq B$ and which is closed under involution, is called a $*$-biideal of $R$.

Motivated by the well-known fact that the algebraic structure of an involution ring is, to a large extent, determined by the algebraic nature of its symmetric or skewsymmetric elements, we study $*$-subdirectly irreducible rings with identity and in which norm elements are central and non-nilpotent. Such involution rings are shown to be rings of the following types: a division ring, a $2 \times 2$ matrix ring over a field, $F \oplus F$ where $F$ is a field, a subdirectly irreducible ring with identity and squarezero heart. Abu-Khuzam [1] studied the structure of certain rings which are multiplicatively generated by their nilpotents. In this note, we shall extend this result to involution rings by conditioning only the norm of the ring. Indeed, we prove that an involution ring $R$ in which the norm is multiplicatively generated by nilpotents is nil if it satisfies the descending chain condition (d.c.c.) on $*$-biideals or if the norm is central and $R$ satisfies the polynomial identity $x^{m}=x^{m+1} f(x)$, where $m$ is a positive integer and $f(x)$ is a polynomial with integer coefficients. 
We recall that an involution ring $R$ is called $*$-subdirectly irreducible if the intersection of all its non-zero $*$-ideals (called the $*$-heart) is non-zero. A routine application of Birkhoff's theorem yields that every involution ring $R$ is a subdirect product of $*$-subdirectly irreducible rings, whence they serve as one source of basic building blocks of all involution rings. Hence the study of $*$-subdirectly irreducible rings is desirable.

In the sequel, for an involution ring $R$, we put $S=\left\{a \in R: a^{*}=a\right\}$, the symmetric elements in $R, K=\left\{a \in R: a^{*}=-a\right\}$, the skew-symmetric elements in $R, T=$ $\left\{a+a^{*}: a \in R\right\}$, the trace of $R, N=\left\{a a^{*}: a \in R\right\}$, the norm of $R$. The center of $R$ is denoted by $Z$. Moreover, following Lim [13], we call $R$ a central trace involution ring if $T$ is contained in the center of $R$.

\section{RESULTS}

The following lemma shall be useful in the proof of the next theorem.

Lemma 1. If $R$ is a *-subdirectly irreducible ring with a non-zero central idempotent, then $R$ has identity.

Proof. Let $e \neq 0$ be a central idempotent in $R$. If $e$ is a symmetric element, then $e$ is the identity of $R$. Indeed, the $*$-ideals $R e$ and $\{r-r e: r \in R\}$ have zero intersection and $R e \neq 0$ and so $\{r-r e: r \in R\}=0$, which implies that $e$ is the identity of $R$. Now suppose $e$ is not symmetric. Then $e e^{*}=0$, for, otherwise, $e e^{*}$ would be the identity of $R$ and then $e e^{*}=e$, contradicting the fact that $e$ is not a symmetric. Likewise it follows that $e^{*} e=0$. Clearly, $e+e^{*} \neq 0$ is an idempotent symmetric element and, arguing as above, we conclude that $R$ has identity $e+e^{*}$.

Theorem 1. Let $R$ be $a *$-subdirectly irreducible ring with a non-zero idempotent. If non-zero trace elements in $R$ are central and non-nilpotent, then $R$ is one of the following types:

(i) a field;

(ii) a division ring with involution, 4-dimensional over its centre and $N \subseteq Z$;

(iii) $F \oplus F$, where $F$ is a field, endowed with the exchange involution;

(iv) the $2 \times 2$ matrix ring over a field $F$, with the symplectic involution, i.e.,

$$
\left(\begin{array}{ll}
a & b \\
c & d
\end{array}\right)^{*}=\left(\begin{array}{cc}
d & -b \\
-c & a
\end{array}\right)
$$

(v) a subdirectly irreducible ring with identity and heart $H=H^{*} \subseteq K, H^{2}=0$ and the additive group of $H$ is either an elementary Abelian p-group or a torsion-free divisible group.

Proof. Let $e$ be an idempotent in $R$. Since the trace elements in $R$ are central, the norm elements in $R$ are also central [13]. If $e$ is symmetric, then it is a norm element and therefore central. If $e$ is not symmetric, then $e+e^{*} \neq 0$ is an idempotent trace element and therefore central. It follows from Lemma 1 that $R$ has identity. 
Now either all elements in the $*$-heart $H$ are square zero and skew-symmetric, or all elements in the $*$-heart are skew-symmetric and $h h^{*}=-h^{2} \neq 0$ for some $h \in H$, or there exists an element $h+h^{*} \neq 0$ for some $h \in H$.

In the first two cases, by [5, Proposition 1.1] and our assumptions on $R$, we see that the $*$-heart $H$ is a minimal ideal of $R$. By [8, Proposition 6.2] the additive group of $H$ is either an elementary Abelian $p$-group or a torsion-free divisible group. Moreover, we claim that $R$ is a subdirectly irreducible ring. Indeed, if $H \cap J=0$ for some non-zero ideal $J$ of $R$ then it is clear that $J \cap J^{*}=0$ and $H \subseteq J \oplus J^{*}$. Hence, any $h \in H$ can be represented uniquely in the form $h=a+b^{*}$ for some $a, b \in J$. Now $h^{*}=a^{*}+b=-a-b^{*}$ and so $a+b=-b^{*}-a^{*} \in J \cap J^{*}$. This implies $b=-a$ and thus $h=a-a^{*}$. Therefore $h a=a^{2}-a^{*} a=a^{2}$. But $(h a)^{*}=a^{*} h^{*}=-h a \in J \cap J^{*}$ so that $a^{2}=0$. Thus $\left(a+a^{*}\right)^{2}=0$ and, by our assumption on $R$, we must have $a^{*}=-a$, which implies $h=2 a \in J$, a contradiction.

In the first case, $R$ is a subdirectly irreducible ring with identity and heart $H=$ $H^{*} \subseteq K, H^{2}=0$.

In the second case, let $0 \neq a=h h^{*}, h \in H$. Then $a R=R a=H$. Since $H$ is a prime ring, $a^{2} R=a(a R)=a H \neq 0$. Moreover, since $a^{2}$ is central, $H=a^{2} R$ and $a=a^{2} b$ for some $b \in R$. Now $a b \neq 0,(a b)^{2}=\left(a^{2} b\right) b=a b$ and $(a b)^{*}=-a b=a b$, whence $a b \in H$ is the identity of $R$. Therefore $H=R$ and $R$ is a primitive ring. Since, for each $a \in R, a^{*}=-a$, we have $a^{2} \in S$ and $T=0$. If there exists $a \in R$ such that $2 a \neq 0$, then $(2 a)^{2}=2 a^{2}+2 a^{2} \in T$ and so $(2 a)^{2}$. But then $2 a R$ is a nil right ideal of $R$, a contradiction with the fact that $R$ is Jacobson-semisimple. Moreover, for any $a, b \in R,(a b)^{*}=-a b$, that is, $b^{*} a^{*}=b a=-a b=a b$. Hence $R$ is a field of characteristic 2 .

We study the third case. If $a=h+h^{*} \neq 0$ for some $h \in H$, then $a^{2} R=H$. Hence $a=a^{2} b$ for some $b \in R$ and it follows that $0 \neq a b$ is an idempotent. Thus the identity of $R$ belongs to $H$, whence $H=R$ and $R$ is $*$-simple. Either $R$ is a simple prime ring or $R=I \oplus I^{*}$ where $I$ and $I^{*}$ are simple prime rings and the only proper nonzero ideals of $R$ are $I$ and $I^{*}$ [5]. If $R$ is a simple prime ring then, by [9, Theorem 7] and [12, Theorem 1], it is either a division ring or a $2 \times 2$ matrix ring over a field, endowed with the symplectic involution. If $R$ is a division ring, then it follows from [6, Theorem 2] that $R$ is either a field or 4-dimensional over its centre. Finally, we show that if $R=I \oplus I^{*}$, then $I$ is a field. In this case, the identity of $R$ is of the form $e+e^{*}$ with $e \in I$. Since $R$ has no non-zero nilpotent trace elements, it is clear that $I$ cannot have non-zero nilpotent elements. Now, for any $x \in R$, ex-exe and $x e-e x e$ are nilpotent elements in $I$ and hence $e x-e x e=x e-e x e=0$, whence $e x=x e$ and $e$ is a central element and so is $e^{*}$. Then $R=R\left(e+e^{*}\right)=\operatorname{Re} \oplus R e^{*}$, $I=R e$, and $e$ is the identity of $I$. In addition, $I$ is commutative. Indeed, if $a, b \in I$, then $\left(a+a^{*}\right)\left(b+b^{*}\right)=\left(b+b^{*}\right)\left(a+a^{*}\right)$, that is, $a b+a^{*} b^{*}=b a+b^{*} a^{*}$. Thus $a b-b a=b^{*} a^{*}-a^{*} b^{*} \in I \cap I^{*}=0$ and $I$ is commutative. Moreover, any $0 \neq c \in I$ is invertible since $c I=I=I c$. 
The following is an example of an involution ring of type (v) in the above theorem.

Example 1. Let $R$ be the set of all $\alpha x+\beta e$ where $\alpha, \beta \in G F(2)$ and $x^{2}=0$, $e^{2}=e, e x=x e=x$. Then $R$ is clearly a commutative ring with identity element and with the identity involution. The only non-zero proper ideal of $R$ is $H=\{\alpha x$ : $\alpha \in G F(2)\}=H^{*}$. Hence $R$ is subdirectly irreducible with identity and heart $H$, where $H^{2}=0$. The trace of $R$ is 0 .

Corollary 1. $R$ is a semiprime $*$-subdirectly irreducible ring with non-zero idempotent and non-zero trace elements in $R$ are central and non-nilpotent if and only if $R$ is one of the following:

(i) a field;

(ii) a division ring with involution, 4-dimensional over its centre and $N \subseteq Z$;

(iii) $F \oplus F$ where $F$ is a field, endowed with the exchange involution;

(iv) the $2 \times 2$ matrix ring over a field $F$, with the symplectic involution.

Corollary 2. Let $R$ be a $*$-subdirectly irreducible ring with identity and with central idempotent norm and trace elements. Then $R$ is one of the following types:

(i) $G F(2)$ with the identity involution;

(ii) $G F(4)$ with the inverse involution;

(iii) $G F(2) \oplus G F(2)$, endowed with the exchange involution;

(iv) the $2 \times 2$ matrix ring over the field $G F(2)$, with the symplectic involution;

(v) a subdirectly irreducible involution ring with identity, of characteristic 2 and heart $H$ in which the elements are symmetric and $H^{2}=0$.

Proof. A division ring $R$ with involution in which every norm element is idempotent has the inverse involution. In fact, for any $0 \neq a \in R, 0 \neq a a^{*}$ and $\left(a a^{*}\right)^{2}=a a^{*}$ implies $a a^{*}=1$ (the identity of $R$ ) and, similarly, $a^{*} a=1$, so that $a^{*}=a^{-1}$. Moreover, since the trace elements are idempotent, the characteristic of $R$ must be 2 . In fact, $(1+1)^{2}=1+1$ implies $1+1=0$. By [7], we know that such a $*$-subdirectly irreducible ring is either $G F(2)$ or $G F(4)$. In $F \oplus F$ ( $F$ a field), $T=N=\{(a, a): a \in F\}$ and hence, if every element in $N$ is idempotent, it is clear that $F \cong G F(2)$. Similarly, in a $2 \times 2$ matrix ring over a field $F$, with the symplectic involution, $T=N=\left\{\left(\begin{array}{cc}a & 0 \\ 0 & a\end{array}\right): a \in F\right\}$ and the idempotence of each element in $T$ implies $F \cong G F(2)$.

Proposition 1. If $R$ is a von Neumann regular involution ring with central idempotent norm elements, then $R$ is isomorphic to a subdirect sum of involution rings $R_{i}$ ( $i \in \Lambda$ ), where each $R_{i}$ is one of the following:

(i) $G F(2)$ with the identity involution;

(ii) $G F(3)$ with the identity involution;

(iii) $G F(4)$ with the inverse involution;

(iv) $G F(2) \oplus G F(2)$, endowed with the exchange involution;

(v) a $2 \times 2$ matrix ring over $G F(2)$, with the symplectic involution. 
Proof. Any nonzero homomorphic image of $R$ is semiprime and has a nonzero central idempotent element. In fact, if $a+I$ is a nonzero element in the factor ring $R / I$, then $a x+I$, where $a x a=a$, is a nonzero idempotent in $R / I$. Let $e+I=$ $a x+I$. If $e e^{*} \notin I$, then $e e^{*}+I$ is a nonzero central idempotent in $R / I$. On the other hand, if $e e^{*} \in I$, then $e+e^{*}+I$ is a nonzero central idempotent in $R / I$. Thus, by Lemma $1, R / I$ has identity and by [13], the norm elements are central if and only if the trace elements are central. Hence, since $R / I$ is von Neumann regular, $R / I$ cannot have nonzero nilpotent trace elements. By Corollary $1, R / I$ is either a division central trace ring, or $F \oplus F$ where $F$ is a field, endowed with the exchange involution, or a $2 \times 2$ matrix ring over a field $F$, with the symplectic involution. If $R / I$ is a division central trace ring, then the characteristic of $R / I$ is either 2 or 3 . Indeed, since the norm elements in $R$ are idempotent, $(1+1)^{4}+I=(1+1)^{2}+I$ (where $1+I$ is the identity of $R / I)$, whence either $(1+1)+I=I$ or $(1+1)^{2}+I=$ $1+I$, that is, the characteristic of $R / I$ is either 2 or 3 . By [7], $R / I$ is of type $G F(2)$, $G F(3)$ or $G F(4)$.

Proposition 2. $R$ is a von Neumann regular involution ring with zero trace and idempotent norm elements if and only if $R$ is a Boolean ring with identity involution.

Proof. We prove the direct implication. For any elements $a, b, c \in R, a^{*}=-a$, $a b=-b a$ and $2 a b c=0$, whence $(2 R)^{3}=0$ and so $2 R=0$. Hence $R$ has characteristic $2, R$ is commutative and $a^{*}=a$. For any $a \in R, a^{4}=a^{2}$ since the norm elements are idempotent. There exists $y \in R$ such that $\left(a^{2}-a\right) y\left(a^{2}-a\right)=a^{2}-a$, that is, $\left(a^{2}-a\right)^{2} y=a^{2}-a$, which implies $a^{2}=a$.

For an involution ring $R$, we let $N^{\prime}$ be the set of symmetric nilpotent elements in $R$ and $E$ the set of symmetric central idempotents in $R$. The proof of the following proposition is as that of [11, Lemma 1], but we include it for the sake of completeness.

Proposition 3. Let $R$ be an involution ring of characteristic 2 having a non-zero central idempotent. Then the following conditions are equivalent:

(i) the symmetric elements in $R$ can be represented as a product of elements in $E \cup N^{\prime}$.

(ii) the symmetric elements in $R$ are central idempotent.

Proof. (i) implies (ii). Suppose symmetric elements in $R$ can be represented as a product of elements in $E \cup N^{\prime}$. Clearly, $R$ has a non-zero central idempotent symmetric element $e$. First, we show that $e N^{\prime}=0$. The $*$-subring $A=e R$ has identity $e$ and its symmetric elements can be represented as a product of elements in $E \cup N^{\prime}$. If $a$ is any element in $N^{\prime}$, then $e+e a=x_{1} x_{2} \cdots x_{n}$ for some $x_{i} \in e E \cup e N^{\prime}$. Since $e+e a$ is invertible in $A, x_{1}$ cannot be nilpotent, so that $x_{1} \in e E$. Hence $e+e a=x_{1}(e+e a)$, which implies $x_{1}=e$ and $e+e a=x_{2} \cdots x_{n}$. Repeating this procedure, we eventually obtain $e+e a=e$, implying $e a=0$. This shows that $e N^{\prime}=0$. Suppose now there exists a symmetric element $r$ in $R$ which cannot be expressed 
as a product of symmetric central idempotents. Let $e+r=y_{1} y_{2} \cdots y_{k}$ for certain $y_{1}, y_{2}, \ldots, y_{k} \in E \cup N^{\prime}$. Since $e N^{\prime}=0$, we have $e=e(e+r)=\left(e y_{1}\right)\left(e y_{2}\right) \cdots\left(e y_{k}\right)$ and the elements $y_{1}, y_{2}, \ldots, y_{k}$ are central idempotent. Hence $y_{i}+e$ is central idempotent. Moreover, for each $i=1,2, \ldots, n, e y_{i}=e$. So, $r=y_{1} y_{2} \cdots y_{k}+e=$ $\left(y_{1}+e\right)\left(y_{2}+e\right) \cdots\left(y_{k}+e\right)$, which is a contradiction. This shows that every symmetric element in $R$ can be written as a product of central idempotents and hence is central idempotent. The converse is trivial.

Abu-Khuzam [1] studied the structure of certain rings which, as semi-groups, are multiplicatively generated by their nilpotents. We now consider certain involution rings in which the norms are central and multiplicatively generated by nilpotents (that is, the norm elements can be written as a product of nilpotents). We call such an involution ring a $*-N$-ring. If $R$ is a $*-N$-ring with identity, then $R=0$ since the identity of $R$ is a norm element. Moreover, any $*$-homomorphic image of a $*-N$-ring is also such a ring.

Theorem 2. If $R$ is a $*-N$-ring with d.c.c. on $*$-biideals, then $R$ is nilpotent.

Proof. Let $J$ denote the Jacobson radical of $R$ and suppose $J \neq R$. It is wellknown that $J^{*}=J$. Since $R / J$ is a semiprime involution ring with d.c.c. on $*-$ biideals, $R / J$ has an identity $e+J$ [2] and, clearly, $e+J=e^{*}+J=e e^{*}+J$. Since $R / J$ is a $*-N$-ring, by the considerations preceding the theorem, $R / J=0$, that is, $R=J$, a contradiction. Hence $R=J$ and $R$ is nilpotent [3].

Let us recall (see [4]) that a $*$-ideal $P$ of an involution ring $R$ is said to be $*$-prime if $A B \subseteq P$ implies $A \subseteq P$ or $B \subseteq P$, where $A$ and $B$ are $*$-ideals of $R$ and $R$ is is $*$-prime if the zero ideal is $*$-prime. Birkenmeier and Groenewald showed that if $P$ is a $*$-prime $*$-ideal which is not a prime ideal, and $X$ is minimal among prime ideals containing $P$, then $P$ is a prime ideal of $X$. In addition, they showed that the intersection of all the $*$-prime $*$-ideals of $R$ coincides with the prime radical of $R$. Hence, if the prime radical of $R$ is 0 , then $R$ is a subdirect product of $*$-prime rings. We shall use these facts in the proof of the next theorem and we shall also require the following lemmas.

Lemma 2 ([1]). If a ring $R$ satisfies the polynomial identity $x^{m}=x^{m+1} f(x)$, then the Jacobson radical of $R$ is nil.

Lemma 3 ([10]). Let $I$ be any non-zero ideal of a ring $R$. Then there exists a homomorphic image $R^{\prime}$ of $R$ containing an isomorphic copy $I^{\prime}$ of $I$ such that $I^{\prime}$ is essential in $R^{\prime}$.

The proof of the following theorem is an adaptation of the corresponding one for rings without involution in [1].

Theorem 3. Let $R$ be $a *-N$-ring which satisfies the polynomial identity $x^{m}=$ $x^{m+1} f(x)$ ( $m$ is a positive integer and $f(x)$ is a polynomial with integer coeffcients). Then $R$ is nil. 
Proof. The Jacobson radical $J=J^{*}$ of $R$ is nil by Lemma 2. Since $R / J$ is a semiprime involution ring its prime radical is 0 and hence, by the considerations preceding the lemmas, the intersection of all $*$-prime $*$-ideals of $R$ is 0 . Hence $R$ is a subdirect product of $*$-prime rings $R_{i}(i \in \Lambda)$. If $R_{i}$ is a prime ring, then as in [1, Theorem 2], it follows that $R_{i}$ has identity and since it is a $*-N$-ring, $R_{i}=0$. Now suppose $R_{i}$ is not a prime ring. We have that $R_{i}=R / K_{i}$ for some $*$-prime *-ideal $K_{i}$ of $R$ which is not a prime ideal. Now if $X_{i}$ is minimal among prime ideals containing $K_{i}$, then $K_{i}$ is a prime ideal of $X_{i}$, that is $X_{i} / K_{i}$ is a prime ring and satisfies the polynomial identity $x^{m}=x^{m+1} f(x)$. Hence, as in [1], it follows that $X_{i} / K_{i}$ has an identity. According to Lemma 3, there exists a homomorphic image $R_{i}^{\prime}$ of $R_{i}$ containing an isomorphic copy $X_{i}^{\prime}$ of $X_{i} / K_{i}$ such that $X_{i}^{\prime}$ is essential in $R_{i}^{\prime}$. Then, since $X_{i}^{\prime}$ has identity, $X_{i}^{\prime}=R_{i}^{\prime}$. Now $R_{i}^{\prime} \cong R / Q_{i}$ for some ideal $Q_{i}$ of $R$. Moreover, if $e+Q_{i}$ is the identity of $R / Q_{i}$, then $e^{*}+Q_{i}=e e^{*}+Q_{i}=$ $n_{1} n_{2} \cdots n_{k}+Q_{i}$ for certain nilpotent elements $n_{1}, n_{2}, \ldots, n_{k}$ of $R$. Suppose $n_{1}$ has degree of nilpotence $t$. Then $n_{1}^{t-1} e e^{*}+Q_{i}=n_{1}^{t-1} n_{1} n_{2} \cdots n_{k}+Q_{i}=Q_{i}$ which implies $n_{1}^{t-1} e^{*} \in Q_{i}$. Therefore $n_{1}^{t-1} e^{*} e n_{2} \cdots n_{k}=n_{1}^{t-2}\left(e^{*} e\right)^{2} \in Q_{i}$. Continuing with this process, we eventually obtain $n_{1}^{t-(t-1)}\left(e^{*} e\right)^{t-1} \in Q_{i}$, that is $n_{1}\left(e^{*} e\right)^{t-1} \in$ $Q_{i}$. Therefore $n_{1}\left(e^{*} e\right)^{t-1} n_{2} \ldots n_{k} \in Q_{i}$ and $\left(e^{*} e\right)^{t} \in Q_{i}$. So, if $t$ is even, $\left(e^{*} e\right)^{t} R=$ $\left(e^{*} e\right)^{\frac{t}{2}} R\left(e^{*} e\right)^{\frac{t}{2}} \subseteq Q_{i}$ and since $Q_{i}$ is a prime ideal of $R,\left(e^{*} e\right)^{\frac{t}{2}} \in Q_{i}$. On the other hand, if $t$ is odd, $\left(e^{*} e\right)^{t+1} R \subseteq Q_{i}$ implies that $\left(e^{*} e\right)^{\frac{t+1}{2}} \in Q_{i}$. Continuing in this way, we eventually obtain $e^{*} e \in Q_{i}$ and hence $e^{*} \in Q_{i}$. Then $e+e^{*}+Q_{i}=e+Q_{i}$ and arguing as above, with $e+e^{*}+Q_{i}$ instead of $e+Q_{i}$, we obtain $e+e^{*} \in Q_{i}$. This implies $R / Q_{i}=0$, which is a contradiction. Thus $R_{i}$ is a prime ring and $R_{i}=0$. It follows that $R / J=0$ and $R=J$ is nil.

\section{REFERENCES}

[1] H. Abu-Khuzam, "A note on rings which are multiplicatively generated by idempotents and nilpotents," Internat. J. Math. Math. Sci., vol. 11, no. 1, pp. 5-8, 1988. [Online]. Available: http://dx.doi.org/10.1155/S016117128800002X

[2] U. A. Aburawash, "Semiprime involution rings and chain conditions," in Contributions to general algebra, 7 (Vienna, 1990). Vienna: Hölder-Pichler-Tempsky, 1991, pp. 7-11.

[3] K. I. Beĭdar and R. Wiegandt, "Rings with involution and chain conditions," J. Pure Appl. Algebra, vol. 87, no. 3, pp. 205-220, 1993. [Online]. Available: http://dx.doi.org/10.1016/ 0022-4049(93)90109-7

[4] G. F. Birkenmeier and N. J. Groenewald, "Prime ideals in rings with involution," Quaestiones Math., vol. 20, no. 4, pp. 591-603, 1997.

[5] G. F. Birkenmeier, N. J. Groenewald, and H. E. Heatherly, "Minimal and maximal ideals in rings with involution," Beiträge Algebra Geom., vol. 38, no. 2, pp. 217-225, 1997.

[6] M. Chacron, "A generalization of a theorem of Kaplansky and rings with involution," Michigan Math. J., vol. 20, pp. 45-54, 1973.

[7] S. Crvenković, I. Dolinka, and M. Vinčić, "On subdirect decomposition and varieties of some rings with involution,” Beiträge Algebra Geom., vol. 43, no. 2, pp. 423-432, 2002. 
[8] H. E. Heatherly, E. K. S. Lee, and R. Wiegandt, "Involutions on universal algebras," in Nearrings, nearfields and K-loops (Hamburg, 1995), ser. Math. Appl. Dordrecht: Kluwer Acad. Publ., 1997, vol. 426, pp. 269-282.

[9] I. N. Herstein and S. Montgomery, "Invertible and regular elements in rings with involution," $J$. Algebra, vol. 25, pp. 390-400, 1973.

[10] G. A. P. Heyman and H. J. le Roux, "On upper and complementary radicals," Math. Japon., vol. 25, no. 3, pp. 327-332, 1980.

[11] Y. Hirano and H. Komatsu, "A characterization of Boolean rings. III," Math. J. Okayama Univ., vol. 27, pp. 33-34, 1985.

[12] Y. Hirano and T. Yanai, "von Neumann regular rings with only finitely many symmetric idempotents," Arch. Math. (Basel), vol. 45, no. 6, pp. 511-516, 1985. [Online]. Available: http://dx.doi.org/10.1007/BF01194890

[13] T. P. Lim, "Some classes of rings with involution satisfying the standard polynomial of degree 4," Pacific J. Math., vol. 85, no. 1, pp. 125-130, 1979. [Online]. Available: http://projecteuclid.org/getRecord?id=euclid.pjm/1102784085

Author's address

D. I. C. Mendes

Departamento de Matematica, Universidade da Beira Interior, Portugal

E-mail address: dmendes@mat .ubi.pt 